\title{
Adiabatic Faraday effect in a two-level Hamiltonian formalism
}

\author{
Basudeb Dasgupta ${ }^{1}$ and Georg G. Raffelt ${ }^{2}$ \\ ${ }^{1}$ CCAPP, The Ohio State University, 191 W. Woodruff Avenue, Columbus, 43210 OH, USA \\ ${ }^{2}$ Max-Planck-Institut für Physik (Werner-Heisenberg-Institut), Föhringer Ring 6, 80805 München, Germany
}

(Dated: 19 June 2010)

\begin{abstract}
The helicity of a photon traversing a magnetized plasma can flip when the $B$-field along the trajectory slowly reverses. Broderick and Blandford have recently shown that this intriguing effect can profoundly change the usual Faraday effect for radio waves. We study this phenomenon in a formalism analogous to neutrino flavor oscillations: the evolution is governed by a Schrödinger equation for a two-level system consisting of the two photon helicities. Our treatment allows for a transparent physical understanding of this system and its dynamics. In particular, it allows us to investigate the nature of transitions at intermediate adiabaticities.
\end{abstract}

PACS numbers: 41.20.Jb, 42.25.Lc, 95.85.Sz, 14.60.Pq

\section{INTRODUCTION}

Different polarization states of electromagnetic waves propagating in media and/or external $B$-fields usually have different refractive indices, leading to a nontrivial evolution of a given polarization state. In astronomy, the rotation of the plane of polarization caused by a magnetized medium along the line of sight (Faraday effect) is the most important example. Such phenomena are perfectly analogous to particle oscillations and, in particular, to neutrino flavor oscillations where the role of polarization is played by flavor. ${ }^{1}$ Moreover, in addition to mixing photons of different polarization, $B$-fields also mix photons with certain other particles, notably gravitons, neutral pions, or hypothetical axionlike particles, which in this context play the role of additional photon polarization states [2].

If the medium (taken to include external fields) varies along the photon trajectory, the refractive indices of two polarization states may cross over. In the adiabatic limit a true crossing is avoided and opposite helicity states are adiabatically connected, leading to complete transformation. In neutrino physics, this phenomenon is known as the Mikheev-Smirnov-Wolfenstein (MSW) effect, allowing for large flavor transformations even when the vacuum mixing angle is small [3 5]. Such resonant transformation effects have also been studied in the context of photon-axion oscillations [2].

However, the case of photons with different polarization has been ignored until recently when Broderick and Blandford [6] for the first time considered the Faraday effect across regions of slow $B$-field reversal where the dispersion relations of photons with opposite helicities cross over. If this transition is adiabatic ${ }^{2}$, photons flip their helicity. The practical astronomical consequence is

\footnotetext{
${ }^{1}$ Optical birefringence can be used to explain neutrino oscillations in a pedagogical demonstration experiment [1].

2 Broderick and Blandford use the term "super adiabatic" in this context, but we prefer to keep the usual terminology.
}

that the usual rotation measure for photon polarization $\mathrm{RM} \propto \int \mathbf{B} \cdot \mathrm{d} \boldsymbol{\ell}$ changes to $\pm \int|\mathbf{B} \cdot \mathrm{d} \boldsymbol{\ell}|$ and thus can build up continuously across regions of opposite field direction. The adiabaticity condition depends on photon frequency. Comparing the Faraday effect at frequencies above and below a certain $\omega_{\text {crit }}$ may allow one to study the geometry of astrophysical magnetic fields.

The main purpose of our paper is to formulate the adiabatic Faraday effect in the more familiar language of particle oscillations using a linearized wave equation. In this way the evolution of photon polarization is described by a Schrödinger equation for the two-level system consisting of the two photon helicity states. While the underlying physics, of course, is precisely as discussed in Ref. [6], our approach helps to bring out the explicit analogy to neutrino oscillations and therefore allows one to borrow both intuition and results from more familiar cases. In addition, we recover the nature of Faraday rotation at intermediate adiabaticity.

The article is organized as follows. In Sec. II we derive a two-level Schrödinger equation for photon polarizations and identify the condition for adiabatic helicity flipping. In Sec. III we work out the impact on linearly polarized states and recover the adiabatic Faraday effect. We also discuss a signature for the transition from adiabatic to non-adiabatic evolution. We conclude in Sec. IV.

\section{HAMILTONIAN APPROACH}

\section{A. Linearizing the wave equation}

The evolution of photon polarization, neutrino flavor oscillations, and similar phenomena derive from the underlying wave equation for the relevant fields. Since dispersion plays a central role, it is easiest to assume harmonic time variation $\mathrm{e}^{-\mathrm{i} \omega t}$ for all fields, i.e. to study the spatial variation of a monochromatic wave. Using natural units with $\hbar=c=1$, one finds a stationary 
Klein-Gordon equation of the form ${ }^{3}$

$$
-\nabla^{2} \mathbf{A}=\left(\omega^{2}-\Pi\right) \mathbf{A}
$$

where $\mathbf{A}$ is a "spinor" of amplitudes describing the multicomponent wave phenomenon. For photon propagation, this is the Jones vector formed by the transverse components of the electric field (Appendix A), whereas for neutrinos the wavefunction in flavor space comes into play. For photons, $\Pi$ is the "polarization tensor," whereas for neutrinos, $\Pi=\mathrm{M}^{2}$ with $M$ being the mass matrix that is non-diagonal in the interaction basis.

The problem simplifies further if the waves are relativistic, i.e. $\omega^{2} \gg\left|m^{2}\right|$, where $m^{2}$ is a typical eigenvalue of $\mathrm{M}^{2}$ or of $\Pi$. In the language of refractive indices, the dispersion relation is written as $k=n \omega$ and the relativistic assumption amounts to $|n-1| \ll 1$. The propagating waves now involve a short length scale (the wave length) and a long one corresponding to the phase difference (the oscillation length). Assuming propagation in the $z$-direction, one can easily separate the fast and slow variation by the nominal substitution [2]

$$
-\left(\partial_{z}^{2}+\omega^{2}\right)=\left(\mathrm{i} \partial_{z}+\omega\right)\left(\mathrm{i} \partial_{z}-\omega\right) \rightarrow-2 \omega\left(\mathrm{i} \partial_{z}+\omega\right),
$$

where we have used $-\mathrm{i} \partial_{z} \rightarrow k \approx \omega$ in one of the terms. This approximation replaces the Klein-Gordon equation with a linear equation

$$
\mathrm{i} \partial_{z} \mathbf{A}=\left(-\omega+\frac{\Pi}{2 \omega}\right) \mathbf{A} .
$$

Moreover, we are not interested in the overall phase of the wave, but only in phase differences between different components. Therefore, on the r.h.s. we may drop terms that are proportional to the unit matrix and find

$$
\mathrm{i} \partial_{z} \mathbf{A}=\mathbf{H} \mathbf{A} .
$$

The "Hamiltonian" is $\mathrm{H}=\Pi / 2 \omega=\mathrm{M}^{2} / 2 \omega$.

Sometimes Eq. (4) is written in terms of a parameter "time," playing the role of an affine parameter along the trajectory. The linear wave equation then manifestly resembles a Schrödinger equation for a two-level system such as a spin evolving in a magnetic field. We stress, however, that Eq. (4) is a classical equation. Using natural units somewhat obscures that $\hbar$ does not appear anyway, at least not when considering the variation of photon polarization along a trajectory.

The analogy to a Schrödinger equation reveals that the linear wave equation provides for unitary evolution along the beam, i.e., any photon that disappears from one polarization state must appear in another. Linearizing the wave equation has removed such physical effects as reflection by inhomogeneities or discontinuities of the

\footnotetext{
${ }^{3}$ We use sans-serif letters to denote matrices in polarization space and bold faced sans-serif letters for "spinors" in this space.
}

medium. In the context of neutrino physics such effects are always vanishingly small, except perhaps near a supernova core, whereas for photons near propagation threshold, polarization-dependent reflection effects may play a nontrivial role.

Likewise, the wave may suffer deflection caused by density variations in the transverse direction. Therefore, the requirement that all polarization components follow the same trajectory with sufficient precision poses nontrivial constraints [6]. Typically, relativistic propagation will be required because near propagation threshold the refractive indices between different components are largest and the differential deflection of different polarization states would be largest.

The connection between a classical two-level equation and a true quantum equation as well as the precise role of the relativistic approximation will be explored elsewhere. Here we simply take advantage of the formal equivalence of our problem with a two-level quantum system.

\section{B. Photon dispersion in magnetized plasma}

Photon dispersion in a cold collisionless plasma with electron density $n_{e}$ and weak external magnetic field $\mathbf{B}$ is determined by the plasma frequency and cyclotron frequency that are respectively ${ }^{4}$

$$
\begin{aligned}
& \omega_{\mathrm{p}}=e \sqrt{\frac{n_{e}}{m_{e}}}=3.7 \times 10^{-11} \mathrm{eV}\left(\frac{n_{e}}{\mathrm{~cm}^{-3}}\right)^{1 / 2}, \\
& \omega_{\mathrm{c}}=e \frac{B}{m_{e}}=1.16 \times 10^{-8} \mathrm{eV}\left(\frac{B}{\text { Gauss }}\right),
\end{aligned}
$$

where the elementary charge $e$ was taken to be positive. The analogous contributions from ions are much smaller and will be neglected.

We decompose $\mathbf{B}$ into a longitudinal component $B_{\|}$ along the direction of propagation ( $z$-direction) and a transverse one $B_{\perp}$. The impact of the magnetic field is determined by the dimensionless parameters

$$
b_{\|, \perp}=\frac{\omega_{\mathrm{c}}}{\omega} \frac{B_{\|, \perp}}{B}=e \frac{B_{\|, \perp}}{\omega m_{e}} .
$$

Propagating modes exist only if (Appendix A)

$$
\omega>\frac{1}{2} \omega_{\mathrm{c}}+\sqrt{\frac{1}{4} \omega_{\mathrm{c}}^{2}+\omega_{\mathrm{p}}^{2}} .
$$

\footnotetext{
${ }^{4}$ In the particle-physics literature, rationalized units with $\alpha=e^{2} / 4 \pi \sim 1 / 137$ are almost always employed, whereas in the context of plasma physics and photon propagation, unrationalized units corresponding to $\alpha=e^{2} \sim 1 / 137$ are used, assuming $\hbar=c=1$ in both cases. We follow the particlephysics tradition and note that a magnetic field of 1 Gauss then corresponds to $1.95 \times 10^{-2} \mathrm{eV}^{2}$. The critical field strength, defined by $\omega_{\mathrm{c}}=m_{e}$, is then correctly found as $B_{\mathrm{crit}}=m_{e}^{2} / e=$ $(0.511 \mathrm{MeV})^{2} / \sqrt{4 \pi \alpha}=8.6 \times 10^{11} \mathrm{eV}^{2}=4.4 \times 10^{13}$ Gauss.
} 
The assumption that we are dealing with relativistic waves thus implies that $\left|b_{\|, \perp}\right| \ll 1$.

The Hamiltonian for the evolution of photon polarization is found to be (Appendix A)

$$
\mathrm{H}=\frac{\omega_{\mathrm{p}}^{2}}{2 \omega}\left(\begin{array}{cc}
b_{\|} & -\frac{1}{2} \mathrm{e}^{-\mathrm{i} 2 \varphi} b_{\perp}^{2} \\
-\frac{1}{2} \mathrm{e}^{\mathrm{i} 2 \varphi} b_{\perp}^{2} & -b_{\|}
\end{array}\right),
$$

where we have dropped terms proportional to the unit matrix. We have used the helicity basis, i.e. the components of the spinor $\mathbf{A}$ are the amplitudes of the two circular polarization components of the wave. The angle $\varphi$ describes the direction of $\mathbf{B}_{\perp}$ relative to a fixed direction transverse to the photon trajectory.

To linear order in $B$, the matrix $\mathrm{H}$ is always diagonal and even if $B$ changes or reverses along the trajectory, nothing new happens. Photons that begin linearly polarized stay that way, except that their plane of polarization rotates by an angle proportional to $\int B_{\|} \mathrm{d} z$. Therefore, we had to include the quadratic terms, responsible for the Cotton-Mouton effect. It is these terms that can flip the photon helicity.

The same conclusion is reached by observing that a longitudinal $B$-field is symmetric relative to rotations around the $z$-direction. Therefore, angular momentum along $z$ is conserved and a longitudinal $B$-field can not induce transitions between photons of opposite helicity. This can be achieved only by the transverse field. By the same token, transverse fields are required to achieve mixing with spin-0 particles such neutral pions or axions or with spin-2 particles such as gravitons.

\section{Adiabatic helicity flip}

If photons propagate in the presence of a purely transverse $B$-field, the Cotton-Mouton effect provides a refractive difference between the modes that are linearly polarized parallel and orthogonal to the field. A photon that is initially circularly polarized will acquire elliptic polarization, eventually reverse its helicity, and later return to the initial polarization in the spirit of an oscillation phenomenon.

However, a realistic situation is different. Astrophysical magnetic fields are not homogeneous and vary in magnitude and direction along any given photon path. In the relativistic limit where $\left|b_{\|, \perp}\right| \ll 1$, the refractive effect of $B_{\|}$is much larger than that of $B_{\perp}$ because the Faraday effect is linear in $B$, the Cotton-Mouton effect quadratic. Therefore, almost everywhere the "mass difference" of photons with opposite helicity is large compared with the mixing energy provided by the transverse field. In other words, the unitary transformation between helicity and propagation eigenstates involves a small mixing angle.

However, $B_{\|}$may vanish somewhere and may reverse while $B_{\perp}$ remains at a typical value. At the $B_{\|}$inversion point the helicity eigenstates become degenerate and their effective masses cross over, except for the small perturbation provided by the transverse field. It prevents an actual crossing of the eigenvalues and leads to an adiabatic helicity reversal in the spirit of the MSW effect.

Within the Landau-Zener approximation [7, 8], the probability for the state to jump over the avoided level crossing while preserving its helicity is

$$
P_{\mathrm{j}}=\mathrm{e}^{-\pi \gamma / 2} .
$$

The adiabaticity parameter $\gamma$ compares the rate-ofchange of the energy splitting with the oscillation frequency. When the unperturbed levels would cross (here the $B_{\|}$reversal), one finds the usual result

$$
\gamma=\left.\frac{4\left|\mathrm{H}_{12}\right|^{2}}{\left|\mathrm{H}_{22}^{\prime}-\mathrm{H}_{11}^{\prime}\right|}\right|_{\mathrm{H}_{22}=\mathrm{H}_{11}}
$$

where a prime denotes $\mathrm{d} / \mathrm{d} z$. In our case this is

$$
\gamma=\frac{\omega_{\mathrm{p}}^{2}}{2 \omega} \frac{b_{\perp}^{4}}{\left|2 b_{\|}^{\prime}\right|}=\frac{\omega_{\mathrm{p}}^{2} \omega_{\mathrm{c}}^{3}}{4 \omega^{4}} \ell_{B},
$$

where we have used that at a $B_{\|}$reversal $B=B_{\perp}$ and therefore $b_{\perp}=\omega_{\mathrm{c}} / \omega$. We have also introduced the length scale of $B$-variation $\ell_{B}^{-1}=\left|B_{\|}^{\prime} / B_{\perp}\right|$. If the magnitude $B$ is fixed so that $B_{\|}=B \cos \theta$ and $B_{\perp}=B \sin \theta$ with $\theta$ the angle between photon direction and $\mathbf{B}$, we find $\ell_{B}=\left|\theta^{\prime}\right|^{-1}$. Moreover, using cycle frequencies $\nu=\omega / 2 \pi$, we find that the upper $\nu$ limit given in Eq. (6) of Ref. [6] corresponds in our treatment to $\gamma=1$. In other words, $\gamma=1$ defines a critical frequency

$$
\omega_{\text {crit }}=\left(\frac{\omega_{\mathrm{p}}^{2} \omega_{\mathrm{c}}^{3}}{4} \ell_{B}\right)^{1 / 4}
$$

such that we are in the adiabatic regime for $\omega \ll \omega_{\text {crit }}$ and in the nonadiabatic regime for $\omega \gg \omega_{\text {crit }}$.

The Landau-Zener approximation is strictly applicable only when $B_{\|}$decreases linearly and certain other conditions are met [9]. There is however substantial literature on its refinements [10]. In particular, the "double exponential" ansatz parameterizes the jump probability in a more widely applicable way [1]. We will continue to use the Landau-Zener formula for its simplicity, but our discussion proceeds unaltered if one substitutes a more accurate expression.

Thus far we assumed that the transverse $B$-field points in a fixed direction, but of course this direction changes on the same length scale $\ell_{B}$ as all other properties. This may have interesting consequences [12]. However, in the adiabatic approximation, $B$-field twisting is slow compared with the oscillation length and thus would not produce new effects. Rapid twisting would lead to nonadiabatic transitions.

The $B$-field may have other variations, for example fast variations caused by turbulence. The evolution of photon polarization in such circumstances could be performed on the level of our Schrödinger equation. Both 
for solar and supernova neutrino oscillations it was found that relatively small density fluctuations of the medium can severely affect the MSW effect [13, 14]. Similar phenomena for radio waves are expected and would be an interesting subject of study.

\section{FARADAY ROTATION}

\section{A. Ordinary Faraday effect}

We now investigate the role of adiabatic helicity flips on the rotation of linearly polarized radiation traversing a region of magnetized plasma. If we begin with $\mathbf{A}_{0}$ at the source, at the detector we will have

$$
\mathbf{A}_{\mathrm{D}}=\mathrm{U}_{\mathrm{tot}} \mathbf{A}_{0}
$$

The unitary matrix taking the initial to the final state is

$$
\mathrm{U}_{\text {tot }}=\mathcal{S} \exp \left(-\mathrm{i} \int_{0}^{\mathrm{D}} \mathrm{Hd} z\right)
$$

where the Hamiltonian in the helicity basis is given by Eq. (9). The parameters $\omega_{\mathrm{p}}, b_{\|, \perp}$ and $\varphi$ all depend on z. At different locations the matrices $\mathrm{H}\left(z_{1}\right)$ and $\mathrm{H}\left(z_{2}\right)$ do not in general commute, so the exponential is understood in the space-ordering convention symbolized by $\mathcal{S}$. Note that we work in the helicity basis. However, if photons are produced and detected in regions that do not involve large transverse fields, the helicity states are identical to the propagation states at source and detector. Thus oscillatory terms depending on the mixing angle at source and detector, as in Ref. [15, 16], will vanish.

In the extreme case when the transverse field $B_{\perp}$ is either zero or so small that it plays no role, $\mathrm{H}$ is always diagonal and one finds explicitly

$$
\mathrm{U}_{\mathrm{tot}}=\left(\begin{array}{cc}
\mathrm{e}^{-\mathrm{i} \phi} & 0 \\
0 & \mathrm{e}^{+\mathrm{i} \phi}
\end{array}\right)
$$

with the phase

$$
\phi=\frac{1}{2 \omega} \int_{0}^{\mathrm{D}} \mathrm{d} z \omega_{\mathrm{p}}^{2} b_{\|}=\frac{e^{3}}{2 \omega^{2} m_{e}^{2}} \int_{0}^{\mathrm{D}} n_{e} \mathbf{B} \cdot \mathrm{d} \boldsymbol{\ell} .
$$

The two helicity states pick up equal but opposite phases, implying that the plane of polarization rotates by the angle $\phi$ : the usual Faraday effect. However, the polarization direction at the source is not known, so the measurable quantity is the variation of $\phi$ with frequency. This "rotation measure" is

$$
\mathrm{RM}=\frac{\phi}{\lambda^{2}}=\frac{e^{3}}{8 \pi^{2} m_{e}^{2}} \int_{0}^{\mathrm{D}} n_{e} \mathbf{B} \cdot \mathrm{d} \boldsymbol{\ell},
$$

where $\omega=2 \pi / \lambda$ has been used, with $\lambda$ being the wavelength. RM itself does not depend on frequency.

\section{B. Adiabatic Faraday effect}

In order to understand the impact of an adiabatic helicity flip we subdivide $U_{\text {tot }}$ into several pieces. To be specific we assume a single $B$-field reversal. The propagation up to somewhat before this point is described by the ordinary Faraday effect. The same is true after the reversal onwards. Therefore, the overall effect is

$$
\mathrm{U}_{\text {tot }}=\mathrm{U}_{2} \mathrm{U}_{\text {flip }} \mathrm{U}_{1}
$$

with

$$
\mathrm{U}_{1,2}=\left(\begin{array}{cc}
\mathrm{e}^{-\mathrm{i} \phi_{1,2}} & 0 \\
0 & \mathrm{e}^{+\mathrm{i} \phi_{1,2}}
\end{array}\right)
$$

The phases are given by ordinary Faraday integrals on path 1, leading from the source to the reversal, and path 2 , leading from the reversal to the detector. If the reversal is non-adiabatic, $U_{\text {flip }}$ is the unit matrix and we recover the previous result: $\phi=\phi_{1}+\phi_{2}$.

On the other hand, if the reversal is perfectly adiabatic it has the effect of exchanging the left- and right-handed helicity states

$$
\mathrm{U}_{\text {flip }}=\left(\begin{array}{ll}
0 & 1 \\
1 & 0
\end{array}\right) .
$$

In general, an additional relative phase arises-we will be more precise later. The flip matrix implies

$$
\mathrm{U}_{\text {tot }}=\left(\begin{array}{cc}
0 & \mathrm{e}^{\mathrm{i}\left(\phi_{1}-\phi_{2}\right)} \\
\mathrm{e}^{-\mathrm{i}\left(\phi_{1}-\phi_{2}\right)} & 0
\end{array}\right) .
$$

If the initial linear polarization state is $\mathbf{A}_{0}=(1,1)$ the non-adiabatic (na) and adiabatic (ad) final states are

$$
\mathbf{A}_{\mathrm{D}}^{\mathrm{na}}=\left(\begin{array}{c}
\mathrm{e}^{-\mathrm{i}\left(\phi_{1}+\phi_{2}\right)} \\
\mathrm{e}^{+\mathrm{i}\left(\phi_{1}+\phi_{2}\right)}
\end{array}\right), \quad \mathbf{A}_{\mathrm{D}}^{\mathrm{ad}}=\left(\begin{array}{c}
\mathrm{e}^{+\mathrm{i}\left(\phi_{1}-\phi_{2}\right)} \\
\left.\mathrm{e}^{-\mathrm{i}\left(\phi_{1}-\phi_{2}\right)}\right)
\end{array}\right) .
$$

The impact of the second part of the trajectory is the same in both cases: the helicity components acquire a relative phase $\phi_{2}$, whereas the impact of the first part is reversed. The overall rotation of linear polarization is

$$
\Phi^{\text {na }}=\phi_{1}+\phi_{2} \quad \text { and } \quad \Phi^{\mathrm{ad}}=-\phi_{1}+\phi_{2} .
$$

In other words, the adiabatic helicity flip has the effect of reversing the rotation measure accrued on the path before the flip. This is physically understood: The helicity states in terms of electric fields are $\left(E_{x} \pm \mathrm{i} E_{y}\right) / \sqrt{2}$. Exchanging helicities amounts to $E_{y} \rightarrow-E_{y}$ and thus reverses the polarization angle relative to the $x$-direction.

We juxtapose the non-adiabatic and adiabatic evolution of RM in Fig. 1 that is analogous to Fig. 2 of Ref. [6]. An important difference is that for the adiabatic case these authors actually show the evolution of the phase difference between the two propagation eigenmodes, a quantity that indeed always increases. However, the only 


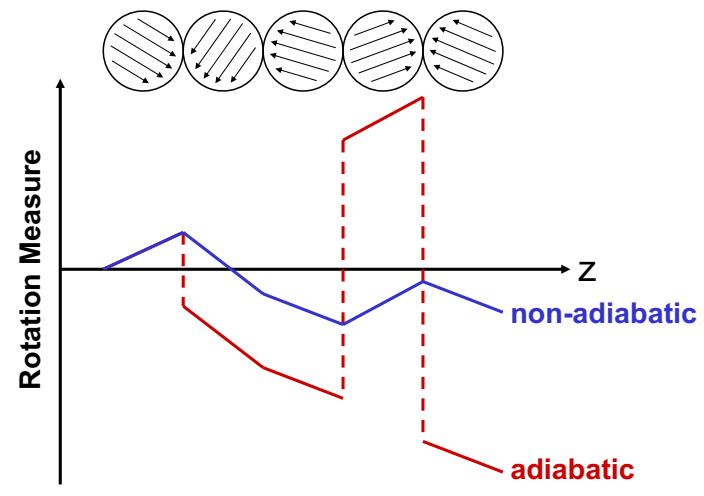

FIG. 1: Evolution of the rotation measure in a magnetized plasma with changing $B$-field orientation. The ordinary (nonadiabatic) and adiabatic cases are shown.

observable quantity is the RM. It acquires a minus sign at each adiabatic reversal and thus jumps by a large amount. The overall adiabatic RM is

$$
\mathrm{RM}^{\mathrm{ad}}= \pm \frac{e^{3}}{8 \pi^{2} m_{e}^{2}} \int_{0}^{\mathrm{D}} n_{e}|\mathbf{B} \cdot \mathrm{d} \boldsymbol{\ell}|
$$

The absolute sign is identical with the sign of $B_{\|}$on the last sub-trajectory.

\section{Intermediate adiabaticity}

The final polarization of the beam depends crucially on the degree of adiabaticity. The entire effect of the reversal is parameterized in the flip matrix. In general, $\mathrm{U}_{\text {flip }}$ is given by a unitary matrix

$$
\mathrm{U}_{\text {flip }}=\mathrm{e}^{\mathrm{i} \alpha}\left(\begin{array}{cc}
\mathrm{e}^{\mathrm{i} \delta_{1}} \cos \xi & \mathrm{e}^{\mathrm{i} \delta_{2}} \sin \xi \\
-\mathrm{e}^{-\mathrm{i} \delta_{2}} \sin \xi & \mathrm{e}^{-\mathrm{i} \delta_{1}} \cos \xi
\end{array}\right)
$$

where $\cos \xi=\sqrt{P_{\mathrm{j}}}$ and $P_{\mathrm{j}}$ is the probability to jump between helicities at the crossing. Note the presence of other phases acquired at the reversal. The non-adiabatic limit is obtained for $\cos \xi=1$ with other phases set to zero. Similarly the adiabatic limit is obtained for $\sin \xi=1$, which is the same as in Eq. (21) for overall phase choices $\alpha=\delta_{2}=\pi / 2$.

If the initial linear polarization state is $\mathbf{A}_{0}=(1,1)$, the final state (up to an overall phase) is

$$
\mathbf{A}_{\mathrm{D}}=\left(\begin{array}{c}
\mathrm{e}^{\mathrm{i}\left(\delta_{1}-\phi_{2}\right)} \cos \xi+\mathrm{e}^{\mathrm{i}\left(\delta_{2}+2 \phi_{1}-\phi_{2}\right)} \sin \xi \\
\mathrm{e}^{-\mathrm{i}\left(\delta_{1}-2 \phi_{1}-\phi_{2}\right)} \cos \xi-\mathrm{e}^{-\mathrm{i}\left(\delta_{2}-\phi_{2}\right)} \sin \xi
\end{array}\right) .
$$

The intensity of the left and right helicity components of this state are

$$
I_{ \pm}=1 \pm \sin 2 \xi \cos \left(\delta-2 \phi_{1}\right)
$$

where $\delta=\delta_{1}-\delta_{2}$ is a combination of the phases acquired at the reversal.
At the fully adiabatic or fully non-adiabatic limit, $\sin 2 \xi=0$ and the two helicities arrive with equal intensity: the beam is linearly polarized. At intermediate adiabaticity this is no longer true and the final beam is elliptically polarized to a degree depending on frequency. Using Eqs. (12) and (13), we find

$$
\cos \xi=\exp \left[-\frac{\pi}{4}\left(\frac{\omega}{\omega_{\text {crit }}}\right)^{4}\right]
$$

whereas from Eq. (16) we find that $\phi_{1}$ varies as $\int \mathrm{d} z \omega_{\mathrm{p}}^{2} b_{\|} / 2 \omega$, the integral extending from the source to the point of reversal.

The degree of elliptical polarization thus has two variations imprinted upon it as a function of frequency. One arises from the usual RM accrued between source and field reversal which determines the orientation with which the linear polarization enters the cross-over region. The other is a slow variation determined by the jump probability as a function of frequency, approximately given by the Landau-Zener formula. If one could measure the polarization state over a reasonably broad range of frequencies, a measure of $B$-field tomography would become possible. Unlike the adiabatic or nonadiabatic limit, at intermediate adiabaticity one has the opportunity to probe the magnetic fields in specific segments of the path traveled.

\section{Multiple reversals}

We have mostly confined our discussion to a single field reversal, but the formalism is easily extended to multiple reversals. As emphasized by Broderick and Blandford, the ordinary Faraday effect for $N \gg 1$ domains, each causing a rotation $\phi_{i}$, adds up to

$$
\Phi^{\mathrm{na}}=\sum_{i=1}^{N} \phi_{i} \propto \sqrt{N} .
$$

On the other hand, the adiabatic Faraday effect leads to a rotation by

$$
\Phi^{\mathrm{ad}}=\sum_{i=1}^{N} \phi_{i} \propto N
$$

Clearly, for media with multiple reversals this leads to a larger Faraday rotation at adiabatic frequencies.

For quasi-adiabatic frequencies, multiple reversals lead to loss of linear polarization by each of the reversals. Schematically, for $N$ domains with $N-1$ field reversals, the intensities of the helicity components arriving at the detector is

$$
\left(\begin{array}{c}
I_{+} \\
I_{-}
\end{array}\right)=\prod_{i=1}^{N-1}\left(\begin{array}{cc}
\cos ^{2} \xi_{i} & \sin ^{2} \xi_{i} \\
\sin ^{2} \xi_{i} & \cos ^{2} \xi_{i}
\end{array}\right)\left(\begin{array}{l}
1 \\
1
\end{array}\right),
$$


where we have ignored the fast oscillatory terms. Clearly, there is increased elliptic polarization at the critical frequencies corresponding to each of the magnetic field reversal. A more precise prediction, including interference effects due to the oscillatory terms, can be calculated using the recipe prescribed in Ref. [17].

\section{CONCLUSIONS}

We have studied the adiabatic Faraday effect that was recently discovered by Broderick and Blandford. We have used a simple formalism that linearizes the photon KleinGordon equation and amounts to a Schrödinger equation for a two-level system consisting of the photon helicities. This approach is commonplace in the context of neutrino flavor oscillations. Once the photon dispersion relation has been identified, the adiabaticity condition follows immediately from well-known textbook results such as the Landau-Zener approximation. Formulating the adiabatic Faraday effect in a language familiar from neutrino physics may allow for a broader appreciation of this intriguing "MSW effect for photons."

Despite the similarities, Faraday rotation also has important differences with neutrino oscillations. For neutrinos, the initial states are always weak-interaction eigenstates, whereas photons can in general be produced in any polarization state. During propagation, neutrinos typically have non-maximal mixing except on the MSW resonance, causing large flavor transitions. For photons, the plane of polarization is typically rotated by many full cycles independently of any transverse field. Therefore, the adiabatic helicity flip does not enhance the transition between linear polarization states, it modifies the rotation measure, the way the rotation of the plane of polarization varies with frequency. Finally, unlike a neutrino detector, a photon detector in the radio band has the capability of identifying not merely the intensities of each polarization state, but also the relative phase between them. So the observational manifestation of the MSW effect is rather different for neutrinos and photons.

To summarize, the adiabatic Faraday effect is the process of photon helicity getting flipped adiabatically in regions of slow $B$-field reversal, and has the effect of reversing the rotation measure accrued by the photon up to that point on its trajectory. Therefore, the subsequent Faraday effect goes effectively in the same direction as the Faraday effect before the reversal region, giving much larger overall rotation measure. The global sign of the rotation measure depends only on the $B$-field direction on the last leg of the photon path.

The rotation measure both in the adiabatic and non-adiabatic regime is independent of frequency. Observations over a broad range of frequencies, ranging from below to above the critical frequency, would reveal a transition range of fast modulations of elliptic polarization. In the adiabatic regime, similar variations can arise in the transverse direction across the sky.

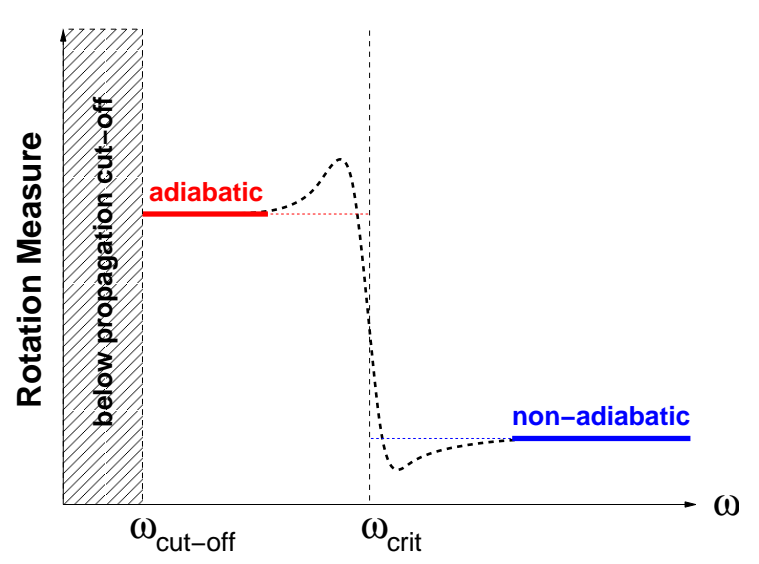

FIG. 2: The rotation measure as a function of frequency. The ordinary (non-adiabatic) and adiabatic regimes are shown.

The astronomical potential of these effects for $B$ field tomography has been explored by Broderick and Blandford. The main purpose of our note was to clarify the basic principles of the adiabatic Faraday effect that is a neat application of the formalism usually applied to the MSW effect in neutrino oscillations.

\section{Note Added in Proof}

After our manuscript had gone to press, D. Melrose circulated a preprint [19], claiming that the adiabatic modification of the Faraday effect was not observable. We believe that underlying this critique is a misunderstanding.

We fully agree with the formalism used by Melrose. Our complex two-spinor $\mathbf{A}$ is equivalent to a $2 \times 2$ density matrix $\mathrm{S}_{i j}=\mathbf{A}_{i} \mathbf{A}_{j}^{*}$ and our Eq. (4) can be written as a commutator equation in the form i $\partial_{z} \mathbf{S}=$ $[\mathrm{H}, \mathrm{S}]$. Moreover, any Hermitean $2 \times 2$ matrix $\mathrm{R}$ can be written in terms of a three-vector $\mathbf{R}$ in the form $\frac{1}{2} \operatorname{Tr}(\mathrm{R})+\frac{1}{2} \mathbf{R} \cdot \boldsymbol{\sigma}$ with $\boldsymbol{\sigma}$ being a vector of Pauli matrices. Our Eq. (4) is equivalent to $\partial_{z} \mathbf{S}=\mathbf{H} \times \mathbf{S}$ where $\mathbf{S}$ is the vector representing $\mathbf{S}$ and $\mathbf{H}$ the one representing H. Therefore, our Eq. (4) is equivalent to the "spinprecession equation" described after Eq. (4) of Ref. [19]. In particular, we agree, that $\mathbf{S}^{2}$ is invariant and that $\mathbf{H} \cdot \mathbf{S}$ is an adiabatic invariant if $\mathbf{H}$ changes slowly as a function of $z$. A precessing spin following a slowly changing $B$-field is the usual visualization of adiabatic neutrino oscillations.

The adiabatic limit is defined by the oscillation length being shorter than the length scale of field reversal, implying that the position angle (PA) of linearly polarized light must undergo many Faraday revolutions between source and detector. Therefore, we agree that PA, being measurable only modulo $\pi$, carries no information about the difference between normal and adiabatic Faraday effect. This information is contained in how quickly PA changes with frequency, i.e. the rotation 
measure (RM). In our Fig. 1 we have sketched how RM varies as a function of distance of a hypothetical observer from the source. However, a real observer is at a fixed location and observes the effect as a function of frequency as sketched in Fig. 2, The observed RM in the normal and adiabatic regime can be different, and are given by Eqs. (18) and (25) respectively. Of course, in the transition region between adiabatic and ordinary Faraday effect, the photon polarization is elliptical. Eq. (28) shows that unless the probability of helicity flip is zero or one, the light is always elliptically polarized. Since the RM is not strictly defined in that case, we sketch the $\mathrm{RM}$ for the major axis of the polarization ellipse.

\section{Acknowledgements}

We thank John Beacom and Leo Stodolsky for inspiring discussions and specific suggestions. This work was partly supported by the Deutsche Forschungsgemeinschaft under grant TR-27 "Neutrinos and Beyond" and the Cluster of Excellence "Origin and Structure of the Universe" (Munich and Garching). B.D. thanks the MaxPlanck-Institut für Physik for support during the initial stages of this work.

\section{Appendix A: Photon dispersion in a cold magnetized plasma}

The propagation of electromagnetic waves in plasma is governed by Maxwell's equations, including polarizations and currents induced by the response to the wave. Assuming a homogeneous cold magnetized plasma, harmonic time variation $\mathrm{e}^{-\mathrm{i} \omega t}$ for all quantities, and spatial variation of the wave only along the $z$-direction, one finds a stationary Klein-Gordon equation for the electric field vector $\mathbf{E}$ of the form [18]

$$
-\left(\begin{array}{lll}
\partial_{z}^{2} & & \\
& \partial_{z}^{2} & \\
& & 0
\end{array}\right) \mathbf{E}=\left(\omega^{2}-\omega_{\mathrm{p}}^{2} \hat{\Pi}\right) \mathbf{E}
$$

where $\omega_{\mathrm{p}}$ is the plasma frequency. $\hat{\Pi}$ is the reduced polarization tensor (polarization tensor in units of $\omega_{\mathrm{p}}^{2}$ ) which depends on $\omega$ and the medium properties.

Assuming the $\mathbf{B}$ field has components $B_{z}=B_{\|}, B_{x}=$ $B_{\perp}$ and $B_{y}=0$ and using the $b$ parameters defined in Eq. (7) one finds

$$
\hat{\Pi}=\frac{1}{1-b_{\|}^{2}-b_{\perp}^{2}}\left(\begin{array}{ccc}
1-b_{\perp}^{2} & \mathrm{i} b_{\|} & -b_{\|} b_{\perp} \\
-\mathrm{i} b_{\|} & 1 & \mathrm{i} b_{\perp} \\
-b_{\|} b_{\perp} & -\mathrm{i} b_{\perp} & 1-b_{\|}^{2}
\end{array}\right) .
$$

The lowest frequencies arise in the homogeneous case when all spatial derivatives vanish and the l.h.s. of Eq. (A1) vanishes identically, leading to

$$
\omega_{0}=\omega_{\mathrm{p}} \quad \text { or } \quad \omega_{0}=\frac{1}{2} \omega_{\mathrm{c}}+\sqrt{\frac{1}{4} \omega_{\mathrm{c}}^{2}+\omega_{\mathrm{p}}^{2}} .
$$

The first solution corresponds to the ordinary plasma oscillation. The other connects to the dispersion relation for propagating modes for non-vanishing $k$ and thus is the minimum frequency required for a propagating wave.

For propagating modes the $z$-equation of Eq. A1 represents a constraint, allowing one to eliminate $E_{z}$. We represent the propagating modes by the usual Jones vector $\mathbf{A}=\left(E_{x}, E_{y}\right)$, leading to a stationary KleinGordon equation in the form

$$
-\partial_{z}^{2} \mathbf{A}=\left(\omega^{2}-\omega_{\mathrm{p}}^{2} \hat{\Pi}\right) \mathbf{A}
$$

The $2 \times 2$ reduced polarization matrix is found to be

$$
\hat{\Pi}_{i j}=\hat{\Pi}_{i j}+\frac{\hat{\Pi}_{i z} \hat{\Pi}_{z j}}{\omega^{2} / \omega_{\mathrm{p}}^{2}-\hat{\Pi}_{z z}}, \quad i, j=x \text { or } y
$$

With the dimensionless parameter $b_{\mathrm{p}}=\omega_{\mathrm{p}} / \omega$ we find for $\hat{\Pi}$ the expression

$$
\frac{1}{\left(1-b_{\mathrm{p}}^{2}\right)\left(1-b_{\|}^{2}\right)-b_{\perp}^{2}}\left(\begin{array}{cc}
1-b_{\mathrm{p}}^{2}-b_{\perp}^{2} & \mathrm{i} b_{\|}\left(1-b_{\mathrm{p}}^{2}\right) \\
-\mathrm{i} b_{\|}\left(1-b_{\mathrm{p}}^{2}\right) & 1-b_{\mathrm{p}}^{2}
\end{array}\right) \text {. }
$$

Notice that the absence of absorption renders this matrix Hermitean.

If all of $b_{\mathrm{p}, \perp, \|}$ are small compared to unity and we expand up to quadratic order we find

$$
\hat{\Pi}=1+b_{\|}^{2}+\left(\begin{array}{cc}
0 & \mathrm{i} b_{\|} \\
-\mathrm{i} b_{\|} & b_{\perp}^{2}
\end{array}\right)+\mathcal{O}\left(b^{3}\right) .
$$

In general the $\mathbf{B}$-field has components $B_{\perp} \cos \varphi, B_{\perp} \sin \varphi$ and $B_{\|}$relative to a coordinate system with a fixed $x-$ direction, implying that the polarization matrix must be rotated correspondingly. Moreover, for some purposes it is simpler to work in the helicity basis where the electric field has the two components $\left(E_{x} \pm \mathrm{i} E_{y}\right) / \sqrt{2}$. Altogether these transformations lead to

$$
\hat{\Pi}=1+b_{\|}^{2}+\frac{1}{2} b_{\perp}^{2}+\left(\begin{array}{cc}
b_{\|} & -\frac{1}{2} \mathrm{e}^{-\mathrm{i} 2 \varphi} b_{\perp}^{2} \\
-\frac{1}{2} \mathrm{e}^{\mathrm{i} 2 \varphi} b_{\perp}^{2} & -b_{\|}
\end{array}\right) .
$$

This is the result used in the main text.
[1] C. Weinheimer, "Neutrino oscillations with a polarized laser beam: an analogical demonstration experiment,"
Prog. Part. Nucl. Phys. 64, 205 (2010).

[2] G. Raffelt and L. Stodolsky, "Mixing of the photon with 
low mass particles," Phys. Rev. D 37, 1237 (1988).

[3] L. Wolfenstein, "Neutrino oscillations in matter," Phys. Rev. D 17, 2369 (1978).

[4] S. P. Mikheev and A. Yu. Smirnov, "Resonance enhancement of oscillations in matter and solar neutrino spectroscopy," Sov. J. Nucl. Phys. 42, 913 (1985) [Yad. Fiz. 42, 1441 (1985)].

[5] H. A. Bethe, "Possible explanation of the solar-neutrino puzzle," Phys. Rev. Lett. 56, 1305 (1986).

[6] A. E. Broderick and R. D. Blandford, "Understanding the geometry of astrophysical magnetic fields," Astrophys. J. 718 (2010) 1085-1099.

[7] L. Landau, "A theory of energy transfer II," Phys. Z. Sowjetunion 2 (1932) 46.

[8] C. Zener, "Nonadiabatic crossing of energy levels," Proc. Roy. Soc. Lond. A 137, 696 (1932).

[9] W. C. Haxton, "Analytic Treatments Of Matter Enhanced Solar Neutrino Oscillations," Phys. Rev. D35 (1987) 2352.

[10] T. K. Kuo and J. T. Pantaleone, "Neutrino oscillations in matter," Rev. Mod. Phys. 61 (1989) 937.

[11] S. T. Petcov, "Exact analytic description of two neutrino oscillations in matter with exponentially varying density," Phys. Lett. B 200 (1988) 373.

[12] J. P. Ralston, P. Jain and B. Nodland, "The corkscrew effect," Phys. Rev. Lett. 81 (1998) 26

[13] C. P. Burgess and D. Michaud, "Neutrino propagation in a fluctuating sun," Annals Phys. 256, 1 (1997)

[14] G. L. Fogli, E. Lisi, A. Mirizzi and D. Montanino, "Damping of supernova neutrino transitions in stochastic shock-wave density profiles," JCAP 0606, 012 (2006)

[15] S. J. Parke, "Nonadiabatic Level Crossing in Resonant Neutrino Oscillations," Phys. Rev. Lett. 57 (1986) 12751278.

[16] S. T. Petcov, "Describing analytically the matter enhanced two neutrino transitions in a medium," Phys. Lett. B406 (1997) 355.

[17] B. Dasgupta and A. Dighe, "Phase effects in neutrino conversions during a supernova shock wave," Phys. Rev. D 75 (2007) 093002

[18] V. L. Ginzburg, The propagation of electromagnetic waves in plasmas (Pergamon, Oxford, 1970).

[19] D. B. Melrose, "Faraday rotation: effect of magnetic field reversals," Astrophys. J. 725 (2010) 1600-1606. 